\title{
Impact of frost temperature and moisture content on frost damage to ceramic bricks
}

\author{
Hans Janssen ${ }^{1, *}$, Chi Feng ${ }^{1}$ and Staf Roels ${ }^{1}$ \\ ${ }^{1}$ KU Leuven, Department of Civil Engineering, Building Physics Section, Kasteelpark Arenberg 40, 3001 \\ Leuven, Belgium.
}

\begin{abstract}
Reliable knowledge on how frost deteriorates building materials is necessary when assessing the hygrothermal performances of building facades. Unfortunately, the current frost-thaw testing methods do not offer dependable knowledge, as they only test at very low temperatures and very high moisture contents, contrary to the spectrum of milder conditions usually met in reality. This paper therefore studies frost damage to four ceramic bricks, with accelerated frost-thaw tests at frost temperatures from $-2{ }^{\circ} \mathrm{C}$ to $-20^{\circ} \mathrm{C}$ and moisture saturation degrees from 0.1 to 1.0 . The ultrasonic method is used to quantify the resulting frost damage, with the Young's modulus reduction as index. The actual frost temperature and moisture content affect the level of frost damage, an effect which is not recognised however in current frost-thaw test protocols.
\end{abstract}

\section{INTRODUCTION}

Frost damage has long been established as one of the most ubiquitous and substantial causes of building material deterioration, yielding reduced mechanical properties (compressive strength, Young's modulus, ...) or actual structural damage (spalling, cracking, ...) [1]. For the correct analysis and design of the frost-thaw resistance of building facades, reliable knowledge on how frost conditions affect building materials is hence required.

At present, the common test methods for frost-thaw resistance usually impose very low temperatures and high moisture contents. Exemplarily, the European Standard EN 12371 for stone and the Technical Specification CEN/TS $772-22$ for brick run at respectively $-10{ }^{\circ} \mathrm{C}$ and $-15^{\circ} \mathrm{C}$ [2-3], both working with samples moisture-conditioned via a period of full immersion. These tests are binary: if the material successfully endures these extreme conditions, it is presumed to perform well in common exterior applications; if not, it is supposed to be inapt for common exterior applications. This binary approach is defensible for newly developed bricks, as it is conservative, but it is often too severe when evaluating renovation projects of existing buildings. For example, thermally retrofitting existing brick facades (through interior or cavity insulation) worsens the frost conditions in the brick, but usually not to the extent as imposed by these frost standards. Therefore, a more realistic evaluation of frost risk at milder conditions - as expected to appear for the considered application - would be more appropriate. Since these milder conditions may equally yield frost damage, it is crucial to perform frost-thaw tests over a spectrum of frost temperatures and moisture contents, instead of solely at extreme conditions hence.

* Corresponding author: hans.janssen@kuleuven.be 
In this paper, we investigate frost damage to four types of ceramic brick, with frost temperatures ranging from $-2{ }^{\circ} \mathrm{C}$ to $-20^{\circ} \mathrm{C}$ and moisture saturation degrees from 0.1 to 1.0 . In the section below, we introduce the ceramic bricks and the experimental details. Then, the results are presented and analysed in detail, with additional attention for their use as damage indicator via an isopleth approach. Finally, the conclusions concerning the impact of frost temperature and moisture content on the frost resistance of ceramic brick materials are drawn.

\section{Materials and methods}

\subsection{Materials}

Four types of ceramic bricks are considered in this investigation. The Vandersanden brick is a frost-resistant brick for exterior applications, whereas the Vogelensangh brick is frost-sensitive and hence limited to interior purposes, both commercially available. In addition, Wienerberger has purposefully produced two types of bricks for this project, with firing temperatures of 925 ${ }^{\circ} \mathrm{C}$ and $970{ }^{\circ} \mathrm{C}$, in an attempt to obtain frost-sensitive bricks.

Table 1 gathers some basic properties: bulk density $\left(\rho_{\text {bulk }}, \mathrm{kg} / \mathrm{m}^{3}\right)$, open porosity $(\phi,-)$, capillary absorption coefficient $\left(\mathrm{A}_{\text {cap }}, \mathrm{kg} / \mathrm{m}^{2} \mathrm{~s}^{0.5}\right)$ and capillary moisture content $\left(\mathrm{w}_{\text {cap }}, \mathrm{kg} / \mathrm{m}^{3}\right)$. Clearly, the Vandersanden brick has a similar bulk density and open porosity as the others, but it differs strongly in its capillary absorption characteristics, reflecting its larger pore size. This may result in a relatively higher frost resistance [1].

In preparation for the frost-thaw testing, the raw bricks are first sawn into uniform samples of $17 \times 10 \times 5 \mathrm{~cm}^{3}$, and then conditioned to the targeted moisture contents, see Section 2.2 .1 below. Subsequently, the moist samples are wrapped with plastic films, to avoid evaporation and hence fluctuations in the moisture content during the tests. They are finally placed vertically in the climate chamber, on top of an insulation board.

Table 1. Basic properties of ceramic bricks

\begin{tabular}{|c|c|c|c|c|}
\hline Brick type & $\boldsymbol{\rho}_{\text {bulk }}$ & $\boldsymbol{\phi}$ & $\boldsymbol{A}_{\text {cap }}$ & $\boldsymbol{w}_{\text {cap }}$ \\
\hline Vandersanden & 1838 & 0.314 & 0.487 & 176 \\
\hline Vogelensangh & 1704 & 0.354 & 0.143 & 244 \\
\hline Wienerberger $\left(925^{\circ} \mathrm{C}\right)$ & 1750 & 0.335 & 0.145 & 248 \\
\hline Wienerberger $\left(970^{\circ} \mathrm{C}\right)$ & 1778 & 0.320 & 0.165 & 229 \\
\hline
\end{tabular}

\subsection{Methods}

\subsubsection{Frost-thaw testing}

Frost-thaw testing on the four bricks is performed for 6 frost temperatures and 7 moisture contents. For a better comparison between the four bricks, these moisture contents are normalised to saturation degrees $(\mathrm{S},-)$, which is the fraction of the pore space occupied by water. The selected frost temperatures and saturation degrees are respectively $-2,-4,-6,-8,-14$ and $-20{ }^{\circ} \mathrm{C}$, and $0.10,0.25,0.40,0.55,0.70 \sim 0.75,0.85$ and 1.0. Given the large number of frost conditions and the limited volume of the climate chamber used, only a single sample of each type of brick is tested at each set of frost temperature and saturation degree. Given the compact nature of the imposed temperature and saturation sets, any deviating outliers can still be detected though. 
The protocol for the actual frost-thaw cycling is based on a now outdated Belgian standard, NBN B05-203 [4], and the imposed temperature profile is shown in Fig. 1. The process starts with a cool down of the ambient air temperature from $15^{\circ} \mathrm{C}$ to $0{ }^{\circ} \mathrm{C}$ within $2 \mathrm{~h}$. After that, the targeted frost temperature is approached at a rate of $-4^{\circ} \mathrm{C} / \mathrm{h}$, and maintained for 8 to $14 \mathrm{~h}$. For the thawing process, the ambient temperature is raised rapidly to $15^{\circ} \mathrm{C}$, in about $1 \mathrm{~h}$, to remain at that level for $6 \mathrm{~h}$. The variable interval at the targeted frost temperature is motivated by the desire for a 24-h cycle in all experiments. In all tests, a sequence of 10 frost-thaw cycles is imposed, with ultrasonic testing after 5 and 10 cycles, see Section 2.2.2 below.

The resultant protocol, and the choices made, can certainly be discussed, as it deviates from other frost-thaw testing approaches in several aspects. However, the disparity between most of the currently available standards is equally sizeable, and no definitive unique methodology is at the moment available. For the relative comparison between brick types and frost conditions intended in this study, the protocol suffices.

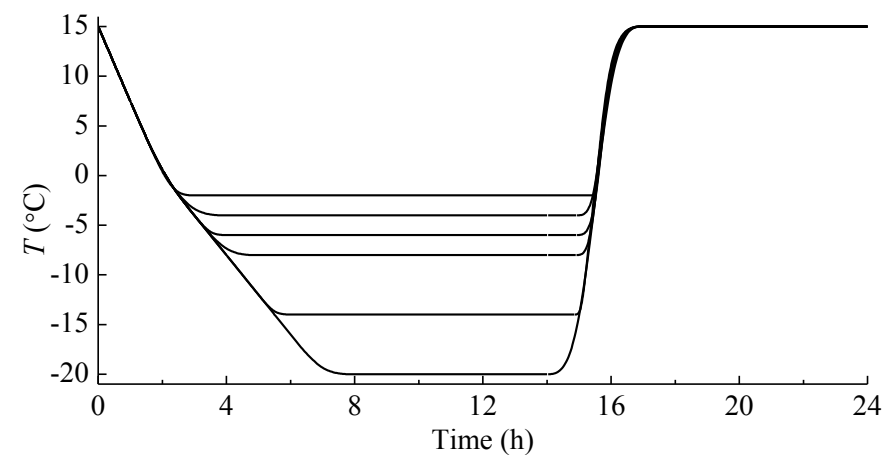

Fig. 1. Temperature profile during the 24-h frost-thaw cycle.

\subsubsection{Ultrasonic testing}

The frost damage is monitored via ultrasonic testing, wherein the state of each sample is judged before the test and after 5 and 10 frost-thaw cycles. For the test, a C373N high performance ultrasonic tester is applied, emitting a pulse with a frequency of $55 \mathrm{kHz}$. The time needed for the pulse to travel across the sample is measured along the longitudinal direction, every time at the same sensor locations. Trial measurements revealed that the plastic film wrapping (to avoid all evaporation) has negligible impact on the experimental results. Similar trial measurements did however also show a substantial variability of the pulse travel time in different samples of the same materials, easily amounting to several 10's of percent, similar to the impact of frost-thaw damage, see Section 3.1 below. Therefore, to obtain reliable findings, it is highly important to compare the same samples before and after testing, instead of using independent sets for before and after tests.

The pulse travel times through the sample are related to the Young's moduli of the materials, and often the relative dynamic modulus (-) is transformed to the damage indicator $\Omega[5,6]$ :

$$
\Omega=1-\frac{E}{E_{0}}=1-\left(\frac{t_{0}}{t}\right)^{2}
$$

where $\mathrm{E}, \mathrm{E}_{0}(\mathrm{~Pa})$ and $\mathrm{t}, \mathrm{t}_{0}(\mathrm{~s})$ are respectively the Young's modulus and the pulse travel time after and before frost-thaw cycling. The evaluation based on Young's modulus is adopted from the standard for natural stone [2]. Other methods prescribe visual evaluation, mass loss quantification or compressive strength testing. Visual inspection is not deemed sufficiently distinctive, no mass loss is expected in the mild condition freeze-thaw cycling, compressive strength tests are complex and expensive, hence the choice for Young's modulus as criterion. 


\section{Results and discussion}

\subsection{Results}

Introductorily, prior to illustrating the effects of the frost temperature and the moisture content on the frost damage, the impact of the number of frost-thaw cycles is exemplified. The ultrasonic test is performed after 5 and 10 cycles, and the comparison of both sets of results is brought in Fig. 2. The graphs reveal that the frost-thaw cycle number has a systematic impact: a larger cycle number always yield larger damage, a reasonable trend that is also found in other studies [1]. Some researchers even claim that frost damage occurs predominantly in the first few frostthaw cycles and is resultantly not heavily affected by the cycle number [7]. This appears to be confirmed by Fig. 2, as generally the impact of the cycle number is quite limited. In what follows, the results after 10 frost-thaw cycles are generally retained.
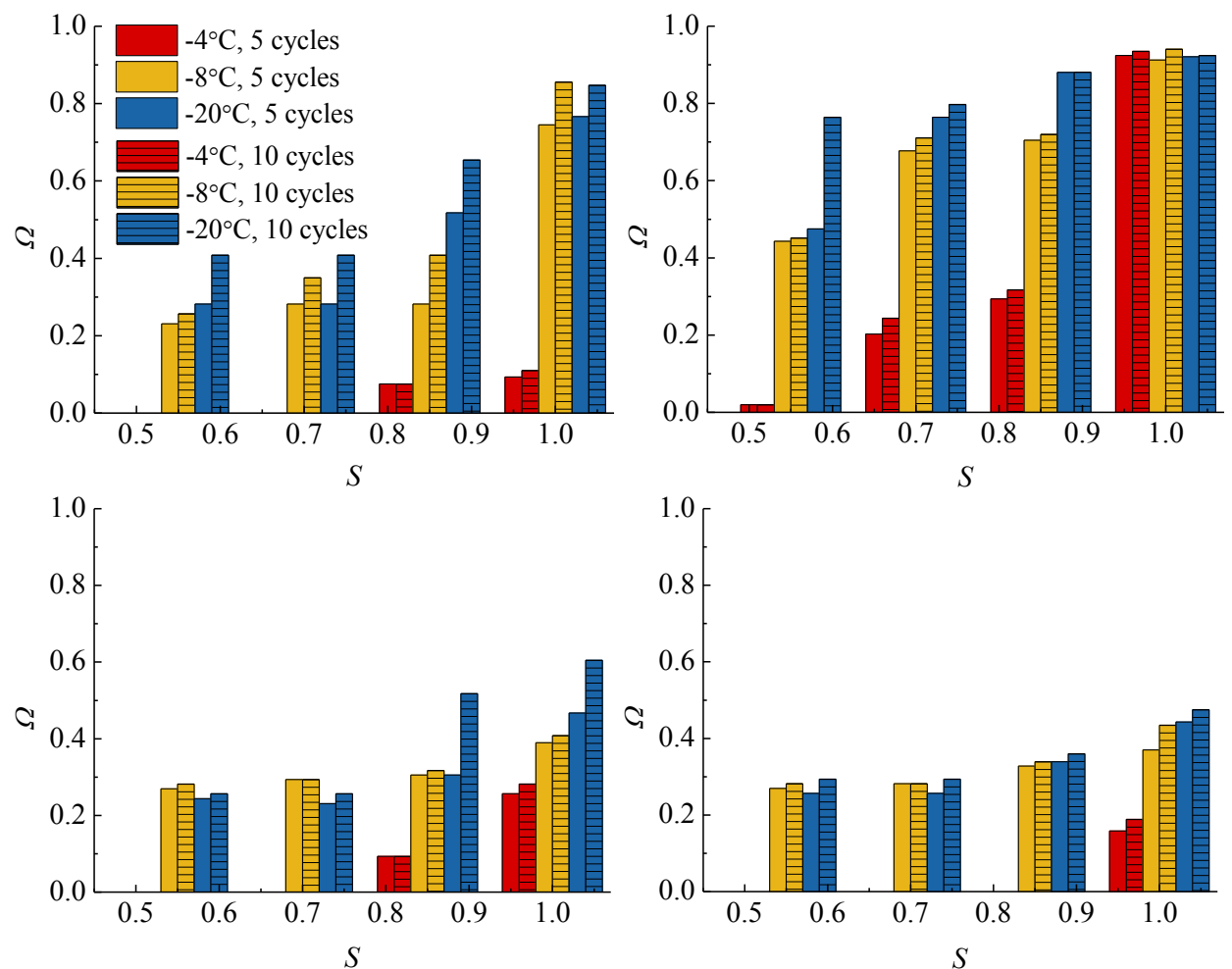

Fig. 2. Influence of the cycle number on the frost damage, for Vandersanden (top left), Vogelensangh (top right), Wienerberger $925^{\circ} \mathrm{C}$ (bottom left), and Wienerberger $975^{\circ} \mathrm{C}$ (bottom right). For reasons of clarity, only selected frost temperatures and saturation degrees are included in these figures. Equal observations result for the other data.

Fig. 3 then finally depicts the influence of the frost temperature and moisture content on the frost damage in the four brick types. The limitation to a single sample for each set of frost temperature and saturation degree leads to some minor irregularities in the damage surfaces, but all in all a reasonably consistent picture is obtained. The figures reveal the Vogelensangh brick to be the most frost sensitive, with extensive damage already at rather mild conditions. The Vandersanden brick is already less sensitive, with extensive damage only at more extreme conditions. The two Wienerberger bricks on the other hand prove to be quite frost resistant, contrary to the intent of their low firing temperatures. 
Overall, lower frost temperatures and higher moisture contents result in more frost damage, as shown by the larger values of $\Omega$. As a result, the most extreme condition $\left(\mathrm{T}=-20^{\circ} \mathrm{C}\right.$ and $\mathrm{S}$ $=1$ ) leads to the strongest frost damage. These extreme conditions are however no predictor of the frost damage at milder conditions. The Vandersanden and Wienerberger $925^{\circ} \mathrm{C}$ bricks are both showing a strong decrease in the frost damage for saturation degrees going down from 1 , whereas the Vogelensangh and Wienerberger $975^{\circ} \mathrm{C}$ bricks have somewhat of a plateau for the higher saturation degrees. Only a complete testing of the full spectrum of possible conditions can hence yield a dependable characterisation of the frost damage sensitivity.
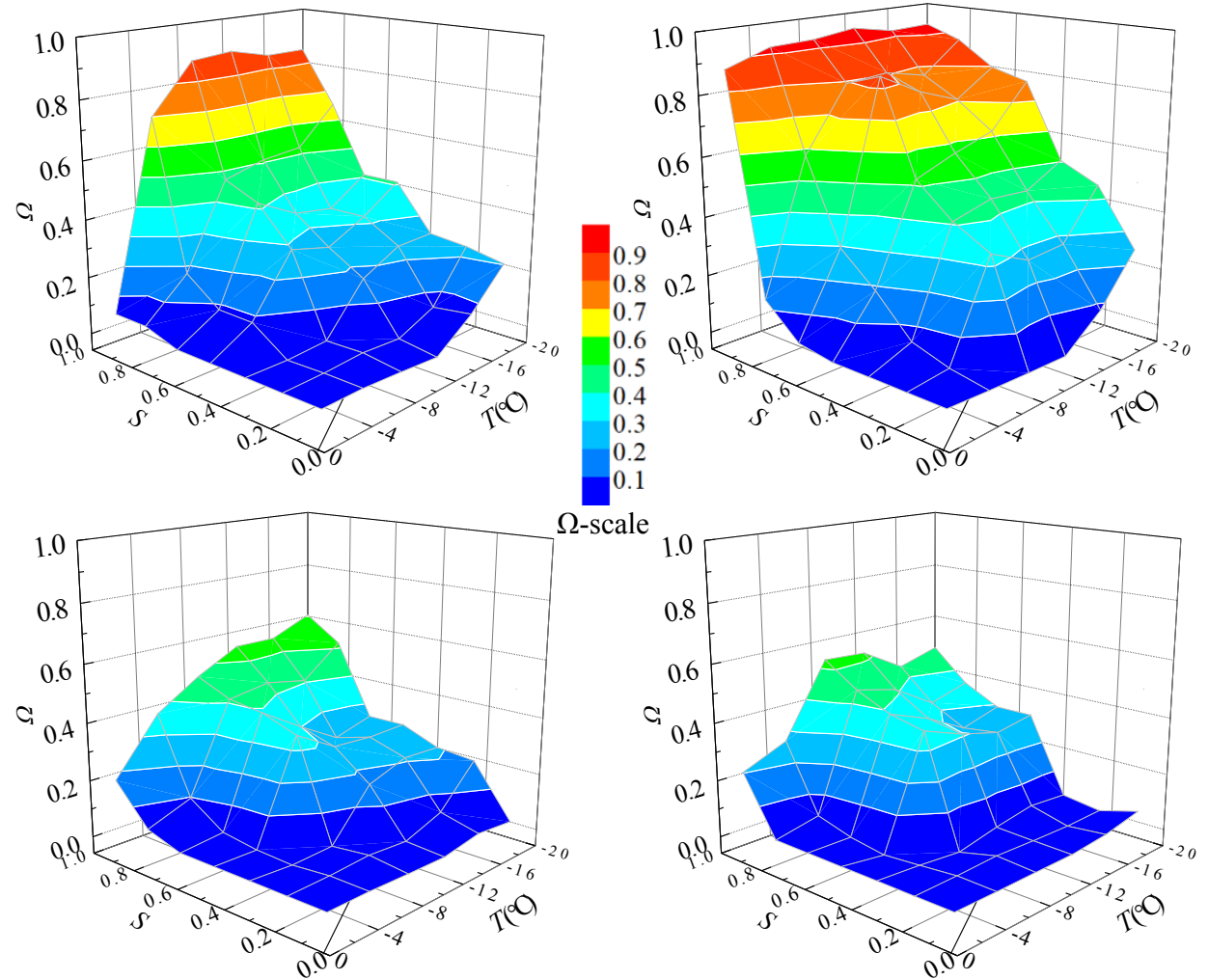

Fig. 3. Influence of the frost temperature $\mathrm{T}$ and saturation degree $\mathrm{S}$ on the frost damage, for Vandersanden (top left), Vogelensangh (top right), Wienerberger $925^{\circ} \mathrm{C}$ (bottom left), Wienerberger $975^{\circ} \mathrm{C}$ (bottom right).

\subsection{Discussion}

Frost damage to building materials has been a central topic in the arena of building physics for decades, and resultantly there currently are multiple damage indicators quantifying existing or future frost damage. Examples are the Modified Winter Index, the Time-of-Frost function, the Amount-of-Frozen-Water or the Number of indicative Freeze-Thaw Cycles [8]. None of these however correctly represent the complex damage relations of Fig. 3, accounting for neither the effect of the frost temperature and moisture content nor the impact of the material's mechanical resistance against frost damage. The results shown in Fig. 3 now allow a new approach for the quantification of frost damage, via an isopleth approach as is commonly used for mould growth assessments. An example for the Vandersanden brick is shown in Fig. 4, whereto the surface plot of Fig. 3 is transformed into a contour plot for easier presentation. 


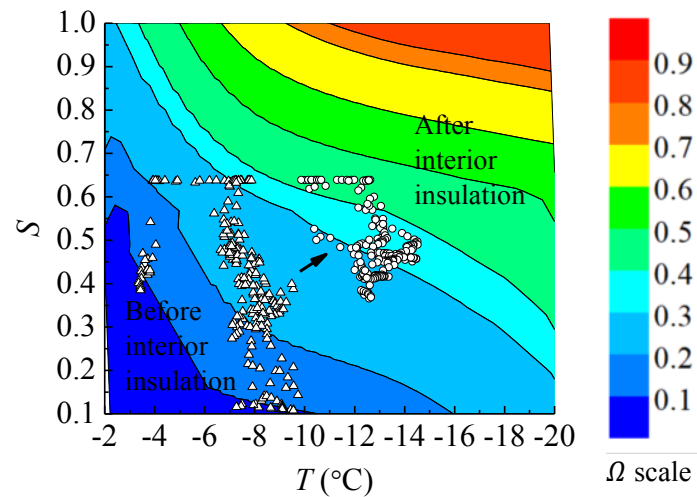

Fig. 4. Contour plot of the $\Omega$ values in function of T and $\mathrm{S}$ for the Vandersanden brick, overlaid with simulated $\mathrm{T}$ and $\mathrm{S}$ results for an (un)insulated massive masonry wall.

The contour plot of the $\Omega$ surface is overlaid with simulated T and $\mathrm{S}$ data for a massive masonry with(out) interior insulation. Without insulation, most of the T-S couples remain below the 0.3 limit [2], indicating only minimal frost damage hence. With insulation, on the contrary, several T-S couples exceed the 0.3 limit, therefore suggesting more severe frost damage.

\section{Conclusions}

This paper has demonstrated that frost-thaw tests at extreme conditions only are insufficient for the dependable evaluation of frost damage under real conditions, which are usually more mild. While such binary testing may suffice for newly developed bricks, the assessment of existing brick materials necessitates frost-thaw tests over a spectrum of frost temperatures and moisture contents, which have exemplarily been performed on four brick types in this study.

This project is supported by EU H2020 project "RIBuild - Robust Internal Thermal Insulation of Historic Buildings" (project No. 637268). We thank Vandersanden, Wienerberger and Vogelensangh, for providing the bricks used in this study. We express our gratitude to Prof. Els Verstrynge, Patricia Elsen and Bernd Salaets from KU Leuven, for supporting the experiments. We acknowledge student Mathias Van Gorp for his contribution to the measurements.

\section{References}

[1] J.P. Ingham, "Predicting the frost resistance of building stone", Quarterly Journal of Engineering Geology and Hydrogeology, vol. 38, pp. 387-399, 2005.

[2] EN 12371:2010: Natural stone test methods - Determination of frost resistance, 2010.

[3] CEN/TS 772-22 Part 22: Methods of test for masonry units - Part 22: Determination of freeze/thaw resistance of clay masonry units, 2006.

[4] NBN B05-203 Resistance of building materials to freezing and thawing cycles, 1977.

[5] ASTM C666/C666M - 15: Standard Test Method for Resistance of Concrete to Rapid Freezing and Thawing, 2015.

[6] K.E. Løland, "Continuous damage model for load-response estimation of concrete", Cement and Concrete Research, vol. 10, pp. 395-402, 1980.

[7] A. Al-Omari, K. Beck, X. Brunetaud, Á. Török, M. Al-Mukhtar. "Critical degree of saturation: a control factor of freeze-thaw damage of porous limestones at the Castle of Chambord, France", Engineering Geology, vol. 185, pp. 71-80, 2015.

[8] J. Kočí, J. Maděra, M. Keppert, R. Černý, "Damage functions for the cold regions and their applications in hygrothermal simulations of different types of building structures", Cold Regions Science and Technology, vol. 135, pp. 1-7, 2015. 